\title{
Understanding replication in a way that is true to science
}

\author{
Brian D. Haig \\ University of Canterbury
}

\begin{abstract}
In this article, I critically examine a number of widely held beliefs about the nature of replication and its place in science, with particular reference to psychology. In doing so, I present a number of underappreciated understandings of the nature of science more generally. I contend that some contributors to the replication debates overstate the importance of replication in science and mischaracterize the relationship between direct and conceptual replication. I also claim that there has been a failure to appreciate sufficiently the variety of legitimate replication practices that scientists engage in. In this regard, I highlight the tendency to pay insufficient attention to methodological triangulation as an important strategy for justifying empirical claims. I argue, further, that the replication debates tend to overstate the closeness of the relationship between replication and theory construction. Some features of this relationship are spelt out with reference to both the hypothetico-deductive and the abductive accounts of scientific method. Additionally, an evaluation of the status of replication in different characterizations of scientific progress is undertaken. I maintain that viewing replication as just one element of the wide array of scientific endeavors leads to the conclusion that it is not as prominent in science as is often claimed.
\end{abstract}

Keywords: nature of science, the status of replication, varieties of replication, methodological triangulation, philosophy of science, reliabilism, phenomena detection, theory construction, scientific method, scientific progress 


\section{Introduction}

The title of this article signals my belief that a good deal of recent methodological work on the topic of replication presupposes an understanding of science that is at variance with what important contemporary science studies reveals about its constitution. By virtue of challenging a number of widely accepted claims about the nature of replication, the article is somewhat contrarian in nature. However, because its content conforms to practices that are commonly found in good science, it is not heterodox.

In the article, I make use of insights in current philosophy of science in order to critically examine a number of widely held beliefs about the nature of replication and its place in science. I do this with particular reference to psychology. However, with few exceptions (e.g., Crandall \& Sherman, 2016; Earp \& Trafimow, 2015; Fidler \& Wilcox, 2017), current methodological research in psychology has made little use of relevant philosophy of science insights in order to better understand replication in science. In a sense, this is not surprising, because modern psychology, more generally, has steadfastly refused to learn from philosophy of science in a systematic way. ${ }^{1}$

Until the 1980s, general philosophy of science largely focused on the nature and role of theory in science. However, the last three decades have witnessed a burgeoning movement, known as the "new experimentalism" (Ackerman, 1989). The movement is visible in the philosophy of physics, and now in the philosophy of biology, but is largely absent from the philosophy of psychology. This is unfortunate, for in its more than 30 years of existence, the new experimentalism has produced a wealth of insights about empirical science, some of which have a direct bearing on how to better understand what replication is, and how it functions in science. Some of the philosophical insights that I draw from are gleaned from the new experimentalism.

In what follows, I argue that some contributors to the replication debates overstate the importance of replication in science, mischaracterize the relationship between direct and conceptual replication, and fail to appreciate sufficiently the variety of legitimate replication practices that scientists engage in. One replication practice I highlight is the tendency to ignore methodological triangulation as an important strategy for justifying empirical claims. I argue, further, that the replication debates tend to overstate the closeness of the relationship between

\footnotetext{
${ }^{1}$ More particularly, psychology makes limited use of philosophy of science in its current meta-scientific research. However, see Devenzer, Navarro, Vanderkerckhove, and Buzbas (2020), who argue the case for employing formal methodology in meta-science and, appropriately, draw from the philosophy of science in doing so. They have instructive things to say about replication practices in science.
} 
replication and theory construction. Some features of this relationship are spelt out with reference to both the hypothetico-deductive and the abductive accounts of scientific method. An evaluation of the status of replication in different characterizations of scientific progress is also undertaken. I maintain that viewing replication as just one element of the wide array of scientific endeavors leads to the conclusion that it is not as central to science as is often supposed.

\section{Two methodological preliminaries}

One of the curious things about scientific discourse is the high frequency with which scientists and methodologists speak of a direct relationship between data and theory (Clark \& Pavio, 1989), even though good research practice often suggests that the relationship is less direct. For good reason, science often deliberately creates a clear methodological space between data and theory, and bridges it with claims about empirical phenomena (Bogan \& Woodward, 1988; Haig, 2013; Woodward, 1989). Briefly, phenomena are stable, recurrent features of the world that we seek to explain. Many of them are noteworthy regularities, and are often called effects. On account of their durability and generality, phenomena serve as the appropriate objects of theoretical explanation. By contrast, data are idiosyncratic to particular investigative contexts, and lack the stability and generality of phenomena. For example, a particular data set of subtest IQ scores cannot be explained by a theory of intelligence. Rather, it is the stable patterns of the positive correlations between subtest scores across different sets of data (the phenomenon of the positive manifold) that constitutes the proper object of theoretical explanation. It should come as no surprise that recent discussions of the nature and role of replication in science also frequently depict the relation between replication and theory construction as a direct one. In evaluating various strands of the replication debates, I repeatedly criticize this tendency to ignore the three-fold distinction between data, phenomena, and theory.

In order to properly understand the place of replication in science, it is also important to distinguish between two fundamentally different types of scientific validation: Reliabilist and coherentist. Reliabilist justification asserts that a belief is justified to the extent that it is acquired by reliable processes or methods (e.g., Goldman, 1986; Goldman \& Beddor, 2017). For example, the appropriate use of a properly calibrated thermometer provides a justification for believing that temperature readings are reliably accurate. By contrast, the coherentist justification of a belief is based on its consistency with other beliefs (e.g., Lycan, 1988). For example, Darwin's belief in the superiority of his theory of natural selection to its creationist 
alternative was largely based on the greater coherence of his theory's constituent beliefs (Thagard, 1992). Importantly, it is reliabilist justification that underwrites claims based on replications, whereas coherentist justification often provides the means to adjudicate between competing theories. It is essential that methodologists and scientists acknowledge these distinct forms of justification, and the different knowledge claims for which they are appropriate. I will be at pains to observe these two distinctions in this article.

\section{The elevated status of replication}

Since antiquity, many eminent thinkers have offered characterizations of the nature of science, and made suggestions for distinguishing science from non-science, and good science from bad science. Here, I confine my attention to two strands of thought: The belief that replication can be employed as a criterion to distinguish science from non-science, and the conviction that it provides the gold standard for justifying scientific claims. I argue that both of these claims elevate the status of replication beyond what can be reasonably justified.

\section{Replication as a demarcation criterion}

Popper (1959) referred to the challenge of distinguishing science from non-science (including pseudo-science), as the problem of demarcation. His well-known solution to this problem was to propose a criterion of falsifiability, which maintains that a theory is scientific if it has the potential to be refuted by empirical observation. A number of philosophers of science have rejected Popper's falsifiability criterion in favor of alternatives (e.g., degree of confirmation, research program progressiveness, problem-solving effectiveness). Others have insisted that a single criterion could not properly do the demarcation work (e.g., Thagard, 1988; Bunge, 1991; Mahner, 2013). Today, there is a broad consensus in philosophy of science that no single criterion can do the job satisfactorily.

The reason for this agreement is quite straight forward: Science itself is highly dimensional in character, with the result that one, or even a few, demarcation criteria fail to properly mark it off from non-science. The multi-faceted nature of science can be quickly appreciated at a general level by noting that it is problem-oriented, pursues multiple aims, employs a wide variety of methods, fashions different sorts of empirical and theoretical claims, and is embedded in institutions that shape its character. More than this, there is considerable variation both within, and across, the different sciences, which thwarts efforts to specify a set of necessary, and sufficient, conditions for demarcation. 
Against this pluralist backdrop, it is surprising that some methodologists in psychology have suggested that replication can serve as a criterion to differentiate science from nonscience. For example, Nosek, Spies, and Motyl (2012) endorse the following claim:

[T] he scientific method differentiates itself from other approaches by publicly disclosing the basis of evidence for a claim ... . In principle, open sharing of methodology means that the entire body of scientific knowledge can be reproduced by anyone. ... Replication is so central to science that it may serve as a "demarcation criterion between science and nonscience." (Braude, 1979, p. 2). (p. 618)

And, in their extended defense of direct replication as an essential practice in psychological research, Zwann et al. (2018) quote, with seeming approval, this early twentieth-century statement about demarcation:

[T]he ability to systematically replicate research findings is a fundamental feature of the research process. Indeed, the idea that observations can be recreated and verified by independent sources is usually seen as a bright line of demarcation that separates science from non-science. (Dunlap, 1926)

Although these quotations are offered more to emphasize the importance of replication to science than to engage in serious discussion of its merit as a demarcation criterion, they nevertheless express a commitment to the view that replication alone suffices to differentiate science from non-science.

In the face of the multi-factorial composition and variability of science, an attractive solution to the problem of demarcation is to regard it as a cluster of activities; that is to say, for a part, or parts, of different sciences, a cluster of relevant features will better characterize a subject matter domain than a set of necessary and sufficient conditions. Similarly, in formulating an adequate conception of demarcation, several relevant criteria together will enable a more realistic, and contextually sensitive, characterization of how to distinguish science from non-science (Mahner, 2013). More specifically, these cluster characterizations should be understood as family resemblances (Irzik \& Nola, 2010). In other words, the various sciences are loosely held together as a group, not by an invariant set of shared characteristics, but by a multitude of partially overlapping and cross-cutting relations. 
Applications of such criteria are allowed to vary from case to case, as the nature of the subject matter demands.

Importantly, replication might be one relevant demarcation criterion in a given instance, but there will undoubtedly be a number of other relevant features to consider as well (e.g., adequacy of measurement, reliability of methods, employment of error elimination procedures, degree of evidential support, robustness of empirical claims, and goodness of theory). Some of these features will frequently be present (e.g., reliability of methods); others less so. Moreover, given that there are ways of validating empirical generalizations, apart from replication, replication itself could never be a necessary condition in a family resemblance characterization of demarcation.

\section{Replication as the gold standard}

Another way of overstating the importance of replication in science is to claim that it is the most important criterion of genuine scientific knowledge, likening it to a gold standard, or the supreme court of science. This is a claim that is often made (see Jasny, Chin, Chong, \& Vignieri, 2011; Hubbard, 2016). The following quotations are typical of such claims:

Replicability ... is the supreme court of the scientific system. (Collins, 1992, p. 19)

Replication ... is considered the scientific gold standard. (Jasny, Chin, Chong, \& Vignieri, 2010, p. 1125)

Replicability is almost universally accepted as the most important criterion of genuine scientific knowledge. (Rosenthal and Rosnow, 1984, p. 9)

Replication is the cornerstone of science. (Simons, 2014, p. 76)

However, as important as replication is to science, I think that the widely held belief that replication provides the best certification of scientific knowledge is too severe. As I will argue later, it could only directly apply to claims that are strongly empirically grounded, such as empirical generalizations. As already intimated, claims about the merits of scientific theories are often appropriately justified by invoking criteria to do with their explanatory goodness. Alternatively, theories might be assessed by employing an augmented hypotheticodeductive testing strategy, where replication might, but need not, be a distant link in the extended chain of reasoning involved (see footnote 4 ).

However, even this delimitation is too stringent because, as noted above, empirical claims might be justified by making use of empirical validation strategies other than 
replication (e.g., the calibration of measuring instruments, and controlling for experimental confounds; Franklin, 1990; Haig, 2014; Woodward, 1989). Later, I will describe the nature of methodological triangulation, and highlight its importance as a strategy for justifying empirical claims. What really matters is that some appropriate reliability, or consistency, test or strategy, is used to validate empirical generalizations (Haig, 2006). Replication ranks among the most important of these strategies, but it is not the only one. As Scholl (2017) succinctly puts it in his discussion of reliability in relation to replication, "In the end, what we should care about is reliability, regardless of the specific means by which we got there." (p.39)

We should also remind ourselves that, for a variety of reasons, not all empirical research is capable of being replicated. For example, massive and/or longitudinal data sets will often make re-collecting data very difficult or impossible. Or, strong spatial and temporal dependencies in some disciplines (e.g., parts of ecology) will rule out the possibility of replication (Fidler, Chee, Wintle, Burgman, McCarthy, \& Gordon, 2017). Further, there are legitimate types of research for which replication is not an appropriate, or major, desideratum. These include forms of research employing methodologies such as participant observation, case study, and event study (Devenzer, Navarro, Vandekerckhove, \& Buzbas, 2020; Leonelli, 2018).

\section{Is repeatability a requirement for good science?}

In the next major section, I will cast doubt on a further claim for the high importance of replication: The contention that direct replication is an essential requirement for validating empirical effects. However, before doing so, I want to comment on the widely endorsed supposition that the repeatability of events is a requirement for good science, and that this requirement, somehow, underscores the importance of replication. This repeatability requirement for replication in science is sometimes justified by appeal to an aspect of Popper's thinking about the nature of scientific objectivity. In particular, the following passage from his work is sometimes presented:

Only when certain events recur in accordance with rules or regularities, as is the case with repeatable experiments, can our observations be tested - in principle - by anyone ... . Only by such repetitions can we convince ourselves that we are not dealing with a mere isolated 'coincidence', but with events which, on account of their regularity and reproducibility, are in principle inter-subjectively testable. $(1959, \mathrm{p} .45)$ 
Popper is surely right to emphasize the importance of intersubjective testing in science; it is, after all, an integral part of our common understanding of scientific objectivity. However, it doesn't follow from this fact that intersubjective testing practices have to take the form of replication. To repeat, what really matters is that some reliable testing strategy is employed to transform beliefs into credentialed knowledge claims.

Moreover, although the regularity of the occurrence of events underwrites replication and other reliabilist validation strategies, some objective practices in science study empirical phenomena without focusing on recurrent events. What is required in such cases is that the phenomena under study are of a form that makes them the appropriate targets of explanation for a given content area. For example, in cognitive science, the primary phenomena to be explained are sometimes taken to be human capacities (e.g., learning, language, memory, and decision making), rather than empirical regularities (Cummins, 2000; van Rooij, \& Baggio, 2020). In such cases, the major empirical task is not to detect the phenomena of interest (we know that they exist), but to specify them in an informative way.

Furthermore, the historical sciences are often interested in explaining single events (or event complexes) rather than empirical regularities - for example, paleontological explanations of the extinction of the dinosaurs in the cretaceous period, or the major political events leading to the outbreak of the Second World War.

The influence of Popper (1959) notwithstanding, I conclude that his forthright assertion that "non-replicable single occurrences are of no significance to science" (p.86), is false.

\section{Varieties of replication}

Historical investigations of the nature and place of replication in science reveal a varied set of practices that are undertaken for different purposes. For example, during the early modern period of history (circa 1600-1800), the following replication practices were prominent (Steinle, 2016; see also Schickore, 2011): The widespread repetition of experiments in order to achieve experimental stability; the replication of the results of others' experiments as the sole basis for confirmation; the conduct of successful replications of empirical findings without appeal to explanatory theories; the replication of empirical studies with theories explicitly in mind; and, the acceptance of the findings of experiments without 
validation by replication. ${ }^{2}$ To a lesser extent, something like this varied set of replication practices can be found in current science, and even to some extent in psychology. However, although today's emphasis on the importance of replication is not new, the methodological discourse on replication in psychology has a considerably narrower focus, sometimes maintaining that the replication of others' studies is the only accepted route to scientific validation. I want to encourage psychologists to think more broadly than this.

In the behavioral sciences, a number of overlapping typologies of replication have been proposed (e.g., Hubbard, 2016; Köhler \& Cortina, 2020; Lykken, 1968; Schmidt, 2016; Sidman, 1960). However, the full range of replication practices included in these typologies is little discussed in the current literature. In an influential article, Schmidt (2009) pared down an array of replication possibilities to just two, which he named direct replication and conceptual replication. Influenced in good part by Schmidt's analysis, many methodologists and researchers today frame their discussions about the nature, and importance, of replication in terms of this two-fold distinction.

As a result of this narrowing of focus, a number of important replication practices have not been given due recognition in the current literature. These include what might be called internal and micro replications. Internal replications adopt the same methods, samples sizes, data analyses, and so on, and are often employed multiple times in the same study or research program. This practice is common in fields such as vision science (Scholl, 2017), and cognitive psychology more generally (Zwaan, et al., 2018). More particularly, microreplications are a form of internal replications, but they are seldom recognized as such. As Guttinger (2019) characterizes them, microreplications are not add-ons to existing studies, but partial replications of findings of other research groups. They are undertaken in order to validate new experimental systems, which themselves are designed to produce further knowledge. As noted at the outset, methodological triangulation is another replication practice that deserves more attention. One immediate consequence of acknowledging the existence of these neglected practices, which are often not identified as replications, is that replication, in one form or another, is probably more prevalent in psychology, and other sciences, than is generally acknowledged.

\footnotetext{
${ }^{2}$ In the history of science, one even finds instances of successful scientific advance that relied on poorly replicated evidence. An instructive example is Bogen's (2001) rich case study of John Jackson's successful nineteenth-century investigations of the organization and function of the human brain. Bogen takes Jackson's work as a challenge to the received view that poorly replicated empirical effects should routinely be regarded as epistemically suspect.
} 


\section{Direct and conceptual replication}

When the replication debates in psychology got underway about a decade ago, it was generally thought that conceptual replications were more important for science than direct replications, with the latter being discouraged and difficult to publish. However, the recent reforms have sought, with some success, to give greater billing to the conduct and publication of direct replications. A major reason for this push has been the claim that without the prior close checks provided by direct replication, claims based on conceptual replication might well be unwitting false discoveries (e.g., Chambers, 2017). Hence the concerted push to restore confidence in psychological science by undertaking more direct replication studies (Nosek, et al., 2012; Zwaan et al., 2018). At the same time, there has been some reaffirmation of the value of conceptual replication (e.g., Crandall \& Sherman, 2016; Lynch et al., 2015; Stroebe \& Strack, 2014).

In the remainder of this section, I discuss the nature and role of direct and conceptual replication in science, and evaluate some of the debates about them.

\section{The limits of direct replication}

Although the contrast between direct and conceptual replication comes in degrees, I will, for present purposes, treat them as two discernably different strategies with different research goals. There is an element of idealization in doing this, but working with a strong contrast does capture a good deal of current replication research practice in psychology. It will also enable me to more readily engage with the current literature on the topic. Briefly stated, a direct replication is undertaken in order to faithfully reproduce the relevant conditions of the original study (e.g., duplicating the protocols, samples, and experimental procedures of the original research). By contrast, a conceptual replication is carried out in order to demonstrate the extent to which results of the original study hold across different methods, treatments, participants, and occasions, where these conditions are deliberately altered in order to establish the generalizability of the original findings. In a later section, I will criticize an odd supplementation of this characterization of constructive replication that is sometimes endorsed in the current literature.

As just noted, much attention has focused on building a case for conducting, and publishing, more direct replication studies in psychology, and various constructive proposals for doing so have been made. A prominent early example of the turn to direct replication is the Open Science large scale reproducibility study (Open Science, 2012). A more recent example is the target article by Zwaan, et al. (2018b), which argued that direct replication 
should be a regular occurrence in mainstream psychological research. I will argue that, although direct replication is a major validation strategy, its importance has sometimes been overstated in the current literature. I will also argue, against some commentators, that conceptual replication is an important, indeed indispensable, way of validating claims about empirical phenomena. In addition, I will outline a research strategy that shows one way in which these two forms of replication might be brought together.

Claims attesting to the importance of direct replication for psychological science are numerous. A clear example is Simons' assertion that, “[D]irect replication by multiple laboratories is the only way to verify the reliability of an effect" $(2014$, p. 76$){ }^{3}$ Another claim in the same vein is that of LeBel and Peters (2011), who state, "Across all scientific disciplines, close [i.e., direct] replication is the gold standard for corroborating the discovery of an empirical phenomenon" (p. 375). Mindful of the fact that replication practices in the history of science have been undertaken for different reasons, I think that claims such as these are open to challenge and, moreover, if accepted, might well distort one's conception of what counts as good scientific practice.

I have four reasons for saying this. First, as already noted, internal replications, including microreplications, are essential features of science, even though they are not add-ons, or external replications, as direct replications have commonly been portrayed. Further, direct replications themselves, are quite often conducted within a single laboratory, and for a good practical reason: They allow a team of researchers to do the needed reliability checks without the considerable time delay involved in validation by outside researchers. Of course, reliability checks by other laboratories is desirable where practicable, but what is most relevant is the quality of the replications, not whether or not they are conducted by separate laboratories (Roediger, 2012).

Second, it is important to understand that replication is only one of a number of ways in which science establishes claims about empirical effects. As I have already noted, empirically investigating equipment, including the calibration of instruments, and controlling for confounding factors in research designs, are others.

Third, partly as a result of the recent heavy focus on direct replication, and partly as the result of the uncritical statisticized nature of that focus, the term effect is typically used to

\footnotetext{
${ }^{3}$ In the same article, Simons qualifies this strong claim by saying, "Direct replication by other laboratories is the best (and possibly the only) believable evidence for the reliability of an effect" (p.76). His statements about the value of direct replication are, in good part, a critical response to Stroebe and Strack's (2014) contention that conceptual replications as tests of theories are more informative than direct replications of empirical effects.
} 
refer to the successful direct replication of the focal empirical findings of an original study an outcome that obviously will have limited generality. This understanding of effects contrasts with the established practice in the physical and biological sciences (and to some extent in psychology), which standardly take empirical phenomena to be the empirical effects of interest. Thus, we have, among innumerable examples, the Compton effect in physics, the Baldwin effect in biology, and the Stroop effect and the Flynn effect in psychology (Haig, 2013; Woodward, 1989)]. Effects like these are empirical phenomena, and are often rightly considered the proper objects of scientific explanation. By their very nature, direct replications cannot give us general effects; they can only furnish us with effects for particular studies and their close replicates. It takes the successful employment of strategies, such as conceptual replication, in order to establish the generality of empirical findings, and thereby deem them scientific effects worthy of the name. Note, that in the passage quoted above, LeBel and Peters (2011) speak of the close, or direct, replication of an empirical phenomenon. However, this way of speaking does not apply to empirical phenomena as they are understood in this article, and science more generally.

Finally, an over-emphasis on the importance of direct replication might detract from appreciating the value of theory construction in science. Consider this quotation, again by Simons (2014), made in the course of promoting the worth of direct replications: "Accumulating evidence for reliable effects is the lasting legacy of science - theories come and go, changing to account for new evidence and making new predictions" (p. 79). Statements like this might reinforce the claim that empirical effects detected by (direct) replication should be accorded greater value in science than theories, on account of their greater durability. Of course, reliable effects don't simply come and go, although they sometimes need to be revised, or even rejected, as a result of phenomena decay (Cronbach, 1975). And, although scientific theories are often highly contested, and frequently undergo conceptual change, it would be wrong to think that they are typically short-lived and, therefore, cognitively inferior to empirical effects. To illustrate with one of many available examples, the general theory of intelligence, originating at the beginning of the $20^{\text {th }}$ century as Spearman's two-factor theory of $g$, has undergone extensive and varied conceptual change for more than 100 years, involving both incorporation and replacement of theory parts. Today, it exists in modified form in a climate of vigorous theoretical pluralism, facing challenges from a number of competing theories. I believe that this is the fate of a good number of our theories in psychology. 


\section{The indispensability of conceptual replication}

Just as direct replication deserves a more prominent place in psychological research than it has customarily received, so the vitally important role played by conceptual replications in science needs to be underscored (Crandall \& Sherman, 2016; Lynch, Bradlow, Huber, \& Lehmann, 2015). Constructive replications are important because they are the major, perhaps primary, means by which we establish the existence of claims about empirical phenomena, (Woodward, 1989; Haig. 2013). As I have stated elsewhere, with constructive replication in mind, "[t]hat phenomena detection accords replication pride of place among its research procedures is perhaps the strongest justification of the importance of replication is science" (Haig, 2014, p. 52). Claims about empirical phenomena are an important form of scientific knowledge in their own right. They are also the major explananda (objects of explanation) upon which scientific theories are built. Further, they form an important class of empirical predictions that can be employed in strong hypothetico-deductive theory testing. The importance of empirical phenomena in science is underscored by the fact that their successful detection is a major scientific achievement, for which Nobel prizes are sometimes awarded.

Despite their frequent use in psychology, conceptual replications have at times been given an unusual characterization in the recent literature. In addition to, and in contrast with, the standard characterization noted above, one sometimes finds clear reference to the direct relevance of theory for conceptual replication. For example, Nosek, Spies, and Motyl (2012) state:

Conceptual replications allow abstraction of the explanation for an effect from the particulars of a given operationalization to the theoretical variable that operationalization attempt to manipulate or assess. ... This is vitally important in science when the constructs of interest are unobservable.... [D]emonstrating the same effect with multiple operationalizations provides confidence in its conceptual interpretation. (p. 619)

The wording of this quotation seems to suggest that, for Nosek et al., conceptual replication is not just concerned with "changing the operationalization of the key elements of the design", but is also directly concerned with evaluating explanatory theories. However, this extension of our common understanding of conceptual replication rides roughshod over the important distinctions between data, empirical phenomena, and explanatory theory described earlier. To repeat, it is the detection of empirical phenomena, with their reliabilist justifications, for which conceptual replications are immediately undertaken. In turn, it is claims about the 
empirical phenomena that are either the predictions involved in strong hypothetico-deductive theory testing, or else the proper target of subsequent explanatory theorizing. ${ }^{4}$

Nosek and Errington (2020a) have recently gone further and claimed that "[t]he purpose of replication is to advance theory by confronting existing understanding with new evidence" (p.3). Their revisionist conceptualization of replication is noteworthy because it explicitly rejects the customary distinctions between direct and conceptual replication as "irrelevant and possibly counterproductive for understanding replication and its role in advancing knowledge" (p. 2). This rejection marks a sharp break with their previous willingness to explicitly accept, and work with, these two forms of replication, as evidenced by their claim that " $[\mathrm{t}]$ ogether they, provide ... confidence in the reproducibility of a finding and the explanation for the finding." (Nosek \& Errington, 2017, p. 2) Nosek and Erington's revised conception of replication is also notable for its de-emphasis of the operational characteristics of a study, and increased emphasis on interpreting possible outcomes.

I think that there are a number of problems with their later proposal. First, Nosek and Errington position their revised conception of replication within a theory testing framework where theories are submitted to empirical assessment via predictive testing. However, as noted earlier, hypothetico-deductive confirmation is just one way of validating knowledge claims in science. As I have already emphasized, there are many research contexts where replication is undertaken in order to detect empirical phenomena without a concern with evaluating theories. Nosek and Errington's rethink of the concept of replication, although cast in very general terms, makes no provision for this important data-to-phenomena move in science.

Second, I think Nosek and Errington's claim that the purpose of replication is to advance theory is just wrong. Consider the following research endeavors: qualitatively confirming hypotheses and theories by hypothetico-deductive reasoning; generating theories through abductive reasoning; developing theories thorough analogical modeling; and, inferring to the best of competing explanatory theories. These are quintessentially theory construction endeavors, and they are often appropriately characterized without reference to replication. Taking license with our common understanding of replication in these ways muddies the

\footnotetext{
${ }^{4}$ Hypothetico-deductive theory testing is an extended process. Briefly stated, it involves moving through the sequence of test theory, auxiliary hypotheses, empirical test predictions (hypotheses), directed empirical studies, and inductive confirmation of the test theory via the intermediary hypotheses. The directed empirical studies might, or might not, involve constructive replication, but their role in the testing process occurs at some remove from the substantive theory being evaluated. Concluding that a failed replication casts direct doubt on tenability of the relevant theory, as some researchers do, fails to acknowledge the complexity of hypothetico-deductive testing.
} 
waters. Nosek and Errington's fundamental error here stems from a failure to sufficiently respect the difference between empirical and theoretical work and the different forms of epistemic justification that they bring with them.

Third, in yet a more recent publication, Nosek and Errington (2020b) bring constructive replication back into their thinking in order to consider the operational characteristics needed to judge whether a replication is successful or not. This should not come as a surprise, given the important need to focus on the procedural aspects of both original and replication studies when conducting and interpreting them.

Finally, in addition to the reservations just expressed, Nosek and Errington's justification for their revisionist proposal also depends on construing standard replication research as boring and unimportant, whereas replication in the service of theory is depicted by them as creative and exciting. Given that empirical research and theory building are fundamentally different types of undertaking, and that empirical researchers and theoreticians mostly have correspondingly different interests and talents, it seems implausible to think that the interests of replicators, and the importance of their work, will vary depending on whether or not it was done with theory in mind. It stands to reason that conducting replications in order to discover empirical regularities is likely to slake the interests of those who are adept at experimental work, whereas constructing theories will appeal those who are more theoretically inclined. Further, the successful detection of scientific discoveries through replication work is greatly valued in science, irrespective of whether it leads to explanatory theorizing, or is involved in predictive theory testing. ${ }^{5}$

\section{How direct and conceptual replication relate}

The question, 'Which is more important, direct or conceptual replication?', is ill-posed. One way in which this can be shown is by presenting a plausible, statistically-oriented, multistage model of data analysis for detecting empirical phenomena in which both forms of replication play a valuable role (Haig, 2005; 2014). This model takes the researcher through a sequence of four stages: Initial data analysis, exploratory data analysis, direct replication, and

\footnotetext{
${ }^{5}$ Machery (2020) has also presented a revisionist proposal for understanding replication. For him, replications should be understood as a process of resampling the elements of an original experiment, which are understood as random factors. He maintains that the idea of a conceptual replication is confused because it cannot differentiate between the different, modified elements of a replication (i.e., treatments, measurements, and settings). However, the frequent successful conduct of conceptual replications straightforwardly shows that researchers are able to do this in practice. It is of more than interest to note that, despite presenting his new perspective on replication, Machery recently chose to conduct a replication study in an orthodox, rather than revisionist, manner (Machery, Grau, \& Pury, 2020).
} 
conceptual replication. The initial screening of the data is undertaken in order to assess its quality. Then exploratory data analysis is conducted with the purpose of identifying potentially interesting patterns in the data. Two confirmatory checks on the stability of the data patterns that emerge from exploratory data analysis follow. The first of these involves a form of direct replication, where computer-intensive resampling methods such as the bootstrap, and cross-validation, are often suitable for use in a just-checking role. The final stage involves carrying out conceptual replications in order to establish the validity of results obtained by direct replication. These four phases of data analysis are concerned respectively with data quality, pattern suggestion, pattern confirmation, and generalization.

Although this model of data analysis features both direct and conceptual replication, science sometime successfully conducts conceptual replications in order to establish robust empirical generalizations without the need to build on direct replication. For example, this was the case in the discovery of the Flynn effect of intergenerational IQ score gains (Flynn, 2009). Working on an existing reliable data base of IQ scores obtained by using different tests on people of different ages in different countries, Flynn established the existence of the effect that bears his name. More than this, the robustness of Flynn's effect was affirmed by a subsequent meta-analysis (Trahan, Stuebing, Hiscock, \& Fletcher, 2014), which confirmed earlier estimates of the Flynn effect and its stability across different countries, age groups, samples, measures, and levels of performance. This meta-analysis demonstrates yet another way in which conceptual replications can be undertaken without first undertaking direct replications. Together, these two examples of successful constructive replication provide further evidence that those who demand direct replications in order to establish empirical effects sometimes ask for too much. ${ }^{6}$

\section{Meta-analysis as conceptual replication}

My comment on the role of meta-analysis in establishing constructive replications deserves some amplification. By virtue of calculating the direction, magnitude, and consistency of effect size estimates across studies in a common domain, meta-analysis can be viewed as a method for detecting general positive effects (Schmidt, 1992). If all goes well, it can advance scientific knowledge through the discovery of empirical regularities or

\footnotetext{
${ }^{6}$ In a strong attack on the worth of conceptual replications, Chambers (2012) claims, among other things, that one cannot replicate a concept. This is obviously correct, just as one cannot replicate a theory (see footnote 4). The term, 'conceptual replication' is a poor replacement for the once-accepted term, 'constructive replication'. However, as noted in its standard characterization given above, constructive replications are in fact concerned with establishing the scope of empirical generalizations.
} 
phenomena. In this descriptive-cum-generalizing role, meta-analysis employs an array of statistical methods in order to help establish the existence of robust empirical regularities and can, therefore, be fairly regarded as an inductive, statistically-oriented approach to constructive replication.

Throughout this article, I have placed considerable store on drawing a strong distinction between the descriptive task of phenomena detection and the explanatory task of theory construction. However, some discussions of the nature of meta-analysis ride roughshod over this distinction. For example, having correctly drawn attention to its primary role as a means of phenomena detection, Schmidt (1993) then proceeds to declare that that it is appropriate to regard the formation of the regularities bequeathed to us by meta-analysis as scientific explanations. However, this stipulation fails to appreciate that empirical regularities often have a life of their own, and are different from the causal factors thought to explain them. ${ }^{7}$ Another example of this conflation of description and explanation is Orlitzky's (2012) claim that meta-analysis is abductive, or explanatory, in nature. However, his belief that metaanalysis supplies the required explanatory information that is embedded in his schematic depiction of abductive reasoning is unconvincing, because he inserts that information into the schema without showing that it is the product of meta-analytic work. In my view, metaanalysis primarily serves non-explanatory ends and, as such, is inductive rather than abductive in character.

Given the ubiquity of meta-analysis as a method of quantitative research synthesis, and its status as a source of constructive replications, it follows that the frequency of replication studies published in psychology must be higher than the very low counts that are commonly reported. On a sanguine note, Schmidt and Oh (2016) argue that because meta-analyses are a form of replication studies, and are conducted on a wide scale, most areas of psychology boast numerous replicated findings. They maintain that because well-conducted metaanalyses exhibit variation in design, measures, methods, and subjects, the convergence of evidence through its resultant triangulation process increases confidence in the claims for generalizability.

Finally, I note that because the studies in meta-analyses will typically comprise a mix of stand-alone primary studies, along with an increasing number of both direct and constructive

\footnotetext{
${ }^{7}$ In saying that empirical regularities can have a life of their own, it should be noted that the credibility of the claims about their acceptability will increase when they are successfully drawn into an a good explanatory theory as the objects of its explanation; they get a confirmational boost from the coherentist justification bestowed on the theory (Haig, 2014).
} 
replication studies, best meta-analytic practice, sometimes with the addition of replication (including meta-analytic) information about the meta-analyses themselves, can result in a nested hierarchy of replication practices that attest to the soundness of review conclusions.

\section{Methodological triangulation as a validation strategy}

As noted at the beginning of this article, methodological triangulation is an important means of validating claims about empirical phenomena that has been given insufficient recognition in the replication debates. I turn to it now.

Consider, first, the idea of robustness. This is a methodological notion that has long been considered important in the various sciences (Wimsatt, 2007). The central idea of robustness is that there have to be multiple independent means for establishing the nature and existence of what is being investigated. It is based on the strong intuition that we are entitled to infer the existence of an object of inquiry that stands up under a variety of different tests. According to Wimsatt's (2007) broad formulation, determining robustness involves "the use of multiple independent means to detect, derive, measure, manipulate, or otherwise to access entities, phenomena, theorems, properties, and other things we wish to study" (p. 27).

Psychologists tend to understand robustness in terms of the more familiar concept of triangulation. In fact, as Wimsatt (2007) points out, the sense of triangulation embodied in Campbell and Fisk's (1959) well-known multitrait-multimethod matrix is quite similar to his conception of robustness. Campbell and Fiske's multitrait-multimethod matrix procedure is a triangulation strategy for investigating the robustness of latent psychological constructs. With this procedure, validation involves obtaining convergent results through the use of independent measuring procedures, along with securing discriminant validity, which serves to check that the invariance across tests, methods, and traits is not a result of their insensitivity to the variables under study. Campbell and Fiske's triangulation procedure is an expression of Campbell's (1969) commitment to multiple operationism, a methodological idea that is to be distinguished from the logical positivist doctrine of definitional operationism.

The current methodological debates about replication have given limited attention to the strategy of methodological triangulation as a means of justifying claims about empirical phenomena (Haig, 2014; Munafò \& Smith, 2018). This is somewhat surprising, given that robustness is one of the distinctive features of claims about empirical phenomena. There are a number of possible reasons why this strategy has received little attention. One reason is that the turn to direct replication brought with it an understandable focus on the duplication of methods, whereas methodological triangulation is concerned with securing differences in 
method. A second reason is that methodological triangulation can be a demanding strategy to implement successfully. For example, implementation of the multitrait-multimethod matrix in the social sciences has generally been poor because of a complex of factors, including underdeveloped theory, and a limited understanding of the factors that affect measurement (Fiske \& Campbell, 1992).

A third reason is the failure to appreciate that methodological triangulation itself can take different forms. At a fundamental level, one form of triangulation employs multiple independent methods in order to detect empirical phenomena (Haig, 2014; Kuorikoski \& Marchionni, 2016), where the reasoning proceeds from data to phenomena. A contrasting form of triangulation employs independent methods in order to validate theories. In this case, the reasoning is about the relation between empirical phenomena and theory. Triangulation in detecting phenomena involves controlling for data-related errors in making causal inferences from data to phenomena, but this does not occur with the triangulation of theories.

It is important to distinguish between these two forms of triangulation, because the targets of investigation, and the forms of inference involved, are different. The triangulation of phenomena typically involves data-to-phenomena inductive inferences to empirical regularities, whereas the triangulation of theories involves theory-to-phenomena hypotheticdeductive, and/or phenomena-to-theory abductive, or explanatory, inferences to claims about the existence and/or nature of a theory's latent causal factors. An example that is often given to illustrate the nature of triangulation applied to scientific theories is Jean Perrin's early twentieth-century argument for the existence of molecules, which was based on stable experimental and calculational determinations of Avogadro's number by numerous different and independent means (Salmon, 1984). Clearly, it is the triangulation of empirical phenomena, not theories, which is the immediate target of replication. Note, that this restricted view of triangulation contrasts with Campbell and Fiske's (1959) strategy of triangulation, which extends to theoretical constructs. It is also more circumscribed than the general sense of triangulation recommended by Munafò and Smith (2018), which seems to apply to both phenomena and theories.

To conclude this section, methodological triangulation is a robustness strategy, and is important because good science prizes well-validated knowledge claims. Empirical phenomena, such as empirical generalizations, are highly valued in science because they constitute an important form of knowledge, as well as being the primary objects of explanation. Taken together, these two features force one to accept that triangulation of phenomena is an indispensable validation strategy in science. Moreover, because 
methodological triangulation employs multiple independent methods, it can fairly be regarded as a restricted form of conceptual replication - restricted in the sense that it deliberately alters methods, but not the other elements of a full conceptual replication (Haig, 2014). Note, however, that direct replication, unlike conceptual replication, is not a method of robustness in the sense employed here, because it secures agreement by straight repetition, rather than by convergence of evidence,

\section{Replication and scientific method}

An odd feature of psychology's methodological landscape is its intense focus on many of the particular research methods it has at its disposal, while at the same time it pays little attention to the nature of scientific method at a more general level. Despite the tendency for researchers to speak of the scientific method, the methodologically pluralist nature of science affords us a number of different theories of scientific method (Nola \& Sankey, 2007). Chief among these are the hypothetico-deductive method, abductive method, and inductive method. In this section of the article, I consider replication in relation to the hypothetico-deductive and the abductive theories of method.

\section{Replication and the hypothetico-deductive method}

For more than 150 years, the hypothetico-deductive method has been employed in science to test hypotheses and theories (Laudan, 1981). According to the standard confirmationist account of this method, the scientist takes a hypothesis or a theory and tests it indirectly by deriving from it one or more observational predictions, which are amenable to direct empirical test. If the predictions are borne out by the data, then that result is taken as a confirming instance of the theory. If the predictions fail to square with the data, then that fact is deemed a disconfirming instance of the theory. This bare-bones description is sometimes enlarged on by specifying relevant initial conditions, and auxiliary hypotheses, that are required in order to facilitate derivation of the predictions (Hempel, 1966; Nola \& Sankey, 2007).

With their fixation on hypothesis and theory testing, many, perhaps most, researchers in psychology subscribe to a gloss on the hypothetico-deductive method. They typically do this by embedding null hypothesis significance testing (NHST) within its compass. However, as Meehl (1967) argued over 50 years ago, letting NHST bear the confirmational burden constitutes a very weak theory testing strategy. It should be to psychology's embarrassment that it continues to test hypotheses and theories in this way. The uncritical reliance on NHST 
is also seen in psychology's penchant for taking statistically significant test outcomes as the major, and sometimes sole, criterion for judging whether a replication study has been performed successfully. This malpractice is exacerbated by employing muddled hybrid versions of NHST that are mistakenly taken to comprise ideas from Fisher and Neyman and Pearson's mature thinking on the topic (Haig, 2017; Mayo, 2018).

Further, it should be pointed out that the absence, or failure, of replication does not thereby threaten the basic form of the hypothetico-deductive method, as Munafò et al. (2017) seem to think. The integrity of the hypothetico-deductive method is preserved by reasoning in accordance with it epistemic and logical strictures. Failure to replicate would only tell against the credibility of the test prediction; it would not threaten the inferential structure of the hypothetico-deductive method itself. Moreover, because there will be cases where reliabilist strategies other than replication speak to the test prediction, one could never justifiably say that replication is an essential feature of the hypothetico-deductive method.

\section{Replication and falsification}

A contrasting account of the hypothetico-deductive method is Popper's $(1959 ; 1963)$ falsificationist construal, in which a strategy of bold conjecture and attempted refutation is employed in order to strongly test theories. Theories that have successfully withstood severe attempts to refute them are said to be corroborated by deductive means, not inductively confirmed, and they are taken to be provisional knowledge of the day.

Despite the defacto use of confirmationist hypothetico-deductive method in psychology, the discipline's normative claims about hypothesis and theory testing are often made out to be falsificationist in nature. Thus, good replication research is often portrayed as attempts to disprove claims that empirical effects exist by submitting them to severe tests. For example, in speaking about the replicability of effects, LeBel, Berger, Campbell, and Loving (2018) declare that "falsifiability is not optional", and they go on to say that "a credible hypothesis is one that has repeatedly survived high-quality, risky attempts at proving it wrong (Lakatos, 1970; Popper 1959; 1969)" (p. 1). However, given psychology's strong preference for weak hypothetico-deductive testing, this Popperian methodological injunction is honored more in the breach than the observance. It is a curious feature of researchers in psychology (and elsewhere) that they frequently reference Popper when talking about scientific method. However, in doing so, they give the misleading impression that Popperian testing is common in psychological research (Uchino, Thoman, \& Byerly, 2010). 
There are two related reasons that help explain the absence of strong Popperian testing in psychology. One has to do with the fact that psychological researchers are, in the main, only superficially acquainted with Popper's theory of science (Earp \& Trafomow, 2015). The other stems from the fact that Popper did not provide scientists with sufficient methodological detail to engage in strong testing (Mulkay \& Gilbert, 1981). An explicit attempt to make good on this second deficiency is Mayo's error-statistical, neo-Popperian formulation of strong tests (Mayo, 1996; Mayo, 2018). The novelty of her account involves employing common frequentist statistical methods in order to identify and correct errors in scientific inference. This is achieved by insisting that their use subserves the all-important requirement that knowledge claims must be severely tested. In Mayo's words, “A claim is severely tested to the extent that it has been subjected to and passes a test that probably would have found flaws, were they present" (Mayo, 2018, p. xii). Mayo's approach is neoPopperian because it focuses on error-elimination through strong tests. However, it differs from Popper's hypothetico-deductivism in two respects: As just noted, it provides the operational means for carrying out strong tests, which Popper failed to do. It also breaks from Popper's insistence that deductive inference is the only legitimate form of inference, and stresses the importance of inductive, content-increasing inference in the hypothesis and theory testing process.

The error-statistical perspective on strong testing contains a number of constructive suggestions for improving psychology's research practices. With respect to replication, I note three of them. First, as already anticipated, the error-statistical practice of testing focal empirical claims in a replication study would employ considerably stronger statistical tests than is currently the case. NHST, which is the primary method of choice, needs to be replaced by a coherent account of statistical significance, one that blends compatible elements of the statistical thought of Fisher, Neyman and Pearson, and Popper, as well as those of the authors of the modern error-statistical perspective (Mayo \& Spanos, 2011). Second, the error-statistical perspective emphasizes the importance of distinguishing between statistical and substantive hypotheses. Tests of significance and other frequentist methods directly assess statistical hypotheses only. Unfortunately, psychological researchers often take the results of statistical methods to have direct implications for substantive hypotheses, without seriously considering the relation between the two. Substantive hypotheses should be the major focus of scientific attention, with statistical hypotheses playing a subservient role. Finally, and commendably, the error-statistical outlook on science employs a hierarchy of models (Mayo, 1996; 2018) - minimally, primary, experimental, and data models - in order 
to structure inquiry. By doing so, it is able to create a theory-data gap and bridge it with an intermediate modeling process. This distinction between model types is not unlike the theory/ phenomena/data distinction deployed in this article.

\section{Replication and abductive method}

Abductive, or explanatory, reasoning plays a major role in scientific research, but it largely goes unnoticed in psychology. Importantly, different species of abductive reasoning can be assembled into a broad abductive theory of method (ATOM) that has two major phases: The initial detection of empirical phenomena, and the subsequent abductive construction of theories to explain the relevant phenomena (for elaboration, see Haig, 2005; 2014). When the process of phenomena detection is concerned with the discovery of empirical generalizations, its basic mode of reasoning can be cast as inductive in nature. The process of phenomena detection is included in ATOM proper because specifying the relevant phenomena in an appropriate way is a requirement for the abductive formation of theories to be possible. In its published formulation, ATOM, presents a replication-centered depiction of the process of phenomena detection (essentially, the four-stage model of data analysis presented earlier). However, it should be made clear that replication is not an essential part of the method. Rather, ATOM should be understood as a framework theory of scientific method into which one can insert more specific methods and strategies that help give it some operational bite. In particular research contexts, alternative reliabilist consistency tests might well replace replication strategies. As with the hypothetico-deductive method, ATOM's use of replication is a contingent feature of its operation, not a necessary part of its way of proceeding.

\section{Replication and scientific progress}

As everyone knows, numerous methodologists and scientists believe, for good reason, that psychology (and allied sciences) faces major disciplinary challenges. However, whether or not it is in the midst of a genuine replication crisis is difficult to gauge. Some who have participated in the replication debates believe that we are in the midst of such a crisis (e.g., 
Pashler \& Harris, 2012 $)^{8}$. However, a small group of dissenting voices claims, in different ways, that the crisis narrative, which portrays published research as unreliable, is unjustified. Fanelli (2018), for example, provides some meta-scientific evidence to support the minority view. I think that there are several reasons for being circumspect about this matter. For one thing, we do not know for sure how frequently replications are carried out. Reported figures for the percentage of studies in psychology identified as replication studies are very low (13\%), although slowly rising (e.g., Makel, Plucker, \& Hegarty, 2012). However, as I have already suggested, they are likely to be higher than this because different forms of replication are often "hidden" in science, sometimes being carried out without being named as replications. Another reason for doubting that we are in the grip of a replication crisis is the fact that considerable scientific progress is being made despite the reported problems with replication (Shiffrin, Börner, \& Stigler, 2018). This seeming paradox can be explained by taking account of factors such as the trial and error nature of science, the prominence of exploratory research, the high number of successful scientific results despite many research failures, and the considerable time often needed to validate findings.

Similarly, we cannot, at present, speak with confidence about the true success rates of attempted replications, simply because the published reported figures vary considerably, ranging from about 35 percent to 85 percent. Further, it seems plausible to suggest that, even at the gross level of its sub disciplines, psychology will reveal different rates of success, with psychophysics tending to be high, social psychology considerably lower, and cognitive psychology somewhere in between (compare respectively, Scholl, 2017; Nosek, et al., 2015; Zwann, et al., 2018a). Moreover, psychologists often disagree on whether findings have been successfully replicated for various reasons, including the fact that multiple replications are typically not undertaken (Hedges \& Schauer, 2019; Maxwell et al., 2015). If we add to this the fact that reliabilist validating strategies, such as methodological triangulation and internal replication, are as important as more recognized forms of replication, and accept the reality that frontier science does not produce immediate success stories, then the veracity of the crisis narrative does not look so convincing.

\footnotetext{
${ }^{8}$ Talk of crises in psychology, and science more generally, is everywhere. Thus it is variously said that we are in the midst of a 'science crisis', a 'credibility crisis', a 'replication crisis', a 'theory crisis', a 'measurement crisis' and more. It is as though psychology is like early-1930s Poland, about which the statistician Jerzy Neyman said, "[W]e have in Poland a terrific crisis in everything. ..." (Reid, 1998; cited in Mayo, 2018, p. 137) Crisis claims might be a useful rhetorical device for mobilizing reforms, and some of them undoubtedly point to genuine problems that need solving. However, in order to be convincing, crisis claims need detailed unpacking, and extensive meta-scientific backing.
} 
A different take on the status of replication, and other malpractices in psychology, is that of Nelson, Simmons, and Simonsohn (2017). For them, there has been a real replication crisis in psychology, but it occurred in the decades before its (re)awakening as a problem circa 2010-2012. These authors claim that there have been dramatic improvements in experimental research in psychology since that time, and that the discipline is now in a period of renaissance. I disagree with this assessment. First, if what I suggest immediately above is plausible, then there might not have been a replication crisis to leave behind. One might also note here that a crisis worthy of the name should have an inflection point, which these authors have not identified. Second, although there have been a good number of valuable methodological reforms proposed and, to some extent implemented, in the last decade, Nelson et al. surely exaggerate the extent of their uptake in declaring that "they are orders of magnitude more common". I note, finally, that because a good number of the reforms are not re-emergent, it is, strictly speaking, a misnomer to speak of them as psychology's "renaissance".

Yet another take on the character of the replication movement is offered by Gelman (2018), who declares that the methodological reforms involving statistics and replication amount to something like a major Kuhnian revolution. ${ }^{9}$ Gelman maintains that the old paradigm (which for him involves the arbitrary collection of data and its analysis in terms of $p$ values), has been replaced by a new paradigm with greater quality control (featuring measurement, within-person comparisons, and multi-level modeling). Leaving aside the question of whether these are in fact rival paradigms, it should be emphasized that science is not paradigmatic in Kuhn's (1970) sense, where research domains are dominated by single paradigms. Rather, it is multi-paradigmatic, which is to say that its subject areas are theoretically and methodologically pluralist. Kuhn has often been used in psychology to justify the misleading idea that the history of its conceptual change is landmarked by successive paradigm replacements. A prominent case in psychology is the popular, but questionable, depiction of the demise of behaviourism, and the rise of cognitivism, circa 1960, as a Kuhnian revolution. By contrast, Braat, Engelen, van Gemert, and Verhaegh's (2020) recent fine-grained sociometric analysis of the "rise and fall" of American behaviorism tells a story of diverse conceptual change.

\footnotetext{
${ }^{9}$ It is relevant to point out here that Spellman's (2015) informative history of the current methodological revolution depicts it in analogy to a political revolution, not a Kuhnian revolution; that is, one that challenges the status quo, but "leaves the same people to function in a differently structured environment".
} 
A more plausible picture of scientific growth than the foregoing depiction is one where multiple paradigms undergo evolutionary change at both the micro- and macro-levels. It is relevant to point out here that, in his later work, Kuhn (2000) modified his earlier conception of scientific revolutions, and associated claims, and presented a more evolutionary conception of scientific progress. For the later Kuhn, talk of revolutions gave way to the idea of developmental episodes, priority was given to smaller-scale paradigms exhibiting exemplars for problem-solving, rather than larger-scale disciplinary matrices, and scientific progress came to be understood as the multiplication of specialities, not the dominance of major paradigms (Nickles, 2017). More in keeping with Kuhn's later position on the evolution of scientific progress is Hull's (1988) extended examination of the social and conceptual development of evolutionary biology and systematics.

On the basis of the foregoing, I conclude that psychology might not be in the midst of a replication crisis; it is difficult to say one way or the other. Further, I do not believe psychology has entered a period of methodological renaissance, nor undertaken a revolutionary paradigm change in our research methods practice. Instead, I believe that it is more accurate to hold that it is in an extended period of heightened methodological reform, a period of reformation during which we have made encouraging gains in our understanding and practice of replication, and other aspects of empirical psychological research.

\section{Summary and conclusion}

In attempting to understand the nature and place of replication in science, I have presented a number of ideas that are not commonly considered in the replication debates. It should be useful, therefore, to restate the main points in capsule form. Before offering a final word, I also make a plea for incorporating philosophy of science in current meta-scientific practice.

\section{Summary}

The claim that replication can serve as a criterion to demarcate science from non-science is unrealistic because it fails to appreciate that shifting sets of criteria are needed to satisfactorily account for the varied and multi-dimensional nature of science. Adopting a multi-criterial view of demarcation could help us improve our understanding of science in at least three ways: Appreciating its multi-factorial nature; heightening our comprehension of science by comparing its features with those of non-science, rather than through direct analysis; and motivating efforts to identify the achievements of good science, as opposed to bad science. Similarly, elevating replication to the status of a gold standard for evaluating 
scientific claims is implausible, because it ignores the fact that other means of reliabilist justification are often employed in establishing empirical generalizations, not to mention the fact that criteria for judging theory goodness are chosen on non-reliabilist grounds.

There is a tendency in both substantive research and methodology to align replication too closely with theory construction. Although replications are often undertaken with theory evaluation in mind, the gap between data and theory must be respected, and properly spanned, by inserting the all-important intermediary process of phenomena detection between them. Replication speaks directly to phenomena detection. Phenomena detection speaks directly to theory construction. Therefore, in understanding science, we should focus more broadly on the nature of validation and adopt different modes of justification for empirical and theoretical claims: Reliabilist justification for empirical claims and hypotheticodeductive and abductive justification for claims about theories. Validation subsumes reliability, which in turn subsumes replication.

Moreover, there is much more to science than detecting empirical phenomena and evaluating explanatory theories (e.g., conducting exploratory research, generating and developing theories before evaluating them in their mature form, applying knowledge in translational science). Thus, the current replication debates speak about a considerably smaller part of science than is typically supposed. For this reason, I maintain that replication is not as central to science as it is often made out to be.

A proper understanding of the nature and place of replication in science requires one to take the major theories of scientific method seriously. Subscription to a neo-Popperian, falsificationist account of hypothetico-deductive testing could help us overcome psychology's penchant for weak empirical hypothesis and theory testing. In addition, psychology needs to recognize that, in a methodologically pluralist environment, the detection of empirical phenomena often precedes the construction of explanatory theories. When this happens, replication will, in the first instance, not be concerned with theory construction at all. In this regard, psychology could enrich its methodological practice by explicitly embracing an abductive theory of scientific method in which the detection of empirical phenomena precedes the construction of theories that might be undertaken to explain them.

Both direct and conceptual replication have important roles to play in science. However, they do not always work in tandem. Sometimes constructive replications alone are sufficient to validate empirical generalizations. On other occasions, neither can be employed, and the adoption of alternative validation strategies will be required. In addition, there exists a variety 
of forms of replication, not just external direct and conceptual replication. They include internal replications, such as microreplications, methodological triangulation, and metaanalysis.

Methodological triangulation is a restrictive form of conceptual replication, and it will often be the strategy of choice for validating claims about empirical phenomena. Further, some forms of replication do not even go by the name of 'replication'. Thus, it makes sense to think of replication as a family resemblance of practices. What its different forms have in common is that they are consistency tests involving reliabilist justificatory strategies.

Regarding claims made about replication in relation to scientific progress, I contend that it is not obvious that we are in a state of genuine crisis, nor are we undergoing a methodological renaissance, nor a paradigm change in the use of our research methods. Instead, we are in a period of heightened methodological reform. We need to go beyond the widespread tendency to say "Adopting replication should lead to greater cumulative science", without unpacking what cumulation might mean. At a general level, a better understanding of science will be had by broadening and enriching our conception of scientific progress. In this regard, articulating the roles played by such notions as problem-solving effectiveness, truth, verisimilitude, and knowledge accumulation deserve our considered attention (Bird, 2016).

\section{Conclusion}

Meta-science, understood as the scientific study of science, has recently been promoted heavily in the methods reform literature, in good part as a response to the so-called replication crisis (Hardwicke, Serghiou, Janiaud, et al., 2020; Ioanides, Fanelli, Drake Dunne, \& Goodman, 2015). In reality, the emergence of this movement continues a long-standing, but unobtrusive, practice in a more self-conscious and prominent way. Today, meta-science is understood as the empirical science of science, and is tasked with furnishing us with empirically based evidence about a host of matters to do with the conduct of psychological research. Valuable though this movement is, it is, in my view, conceived too narrowly. For one thing, it limits itself to the use of quantitative research methods in its studies. For another, it pays limited attention to the various science studies disciplines that are dedicated to helping us better understand science. In particular, it makes little use of the philosophy of science as a source of understanding the scientific processes it studies. These days, most philosophers of science view their discipline naturalistically; that is, as a substantive domain that employs the research findings and investigative means of the various sciences in order to study science itself. Viewed in this light, philosophy of science can be considered part of science, including 
psychology, where it is appropriately positioned to offer valuable insights about science itself (Haig, 2011). If meta-science is to fulfill its interdisciplinary aspirations, then it needs to take the philosophy of science seriously.

In the last decade, the concerted focus on replication in the methods reform literature has significantly increased our understanding of how psychology can improve its replication practices. My hope is that this article will, in complementary fashion, help further our understanding of the conceptual foundations of replication and its place science. 


\section{Acknowledgements}

I thank David Funder, Daniel Lakens, Bob Read, and Fran Vertue for helpful comments on an earlier draft of this manuscript. 


\section{References}

Ackerman, R. (1989). The new experimentalism. British Journal for the Philosophy of Science, 40, 185-190.

Bird, A. (2016). Scientific progress. In P. Humphreys (Ed.), The Oxford handbook of philosophy of science (pp. 544-563). Oxford University Press.

Bogen, J. (2001). 'Two as good as a hundred': Poorly replicated evidence in some nineteenth-century neuroscientific research. Studies in History and Philosophy of Science: Biological and Biomedical Sciences, 32, 491-533.

Bogen, J., \& Woodward, J. (1988). Saving the phenomena. Philosophical Review, 97, 303352.

Braat, M., Engelen, J., van Gemert, T., \& Verhaegh, S. (2020). The rise and fall of behaviorism: The narrative and the numbers. History of Psychology, 23, 252-280.

Bunge, M. (1991). What is science? Does it matter to distinguish it from pseudoscience? A reply to my commentators. New Ideas in Psychology, 9, 245-283.

Campbell, D. T., \& Fiske, D. W. (1959). Convergent and discriminant validation by the multitrait-multimethod matrix. Psychological Bulletin, 56, 85-105.

Chambers, C. (2012). You can't replicate a concept. http://neurochambers.blogspot.com/2012/03/you-cant-replicate-concept.html

Chambers, C. (2017). The seven deadly sins of psychology: A manifesto for reforming the culture of scientific practice. Princeton University Press.

Clark, J. M., \& Paivio, A. (1989). Observational and theoretical terms in psychology: A cognitive perspective on scientific language. American Psychologist, 44, 500-512.

Cooper, H., Hedges, L. V., \& Vallentine, J. C. (2019). Research synthesis as a research process. In H. Cooper, L. V. Hedges, \& J. C. Vallentine (Eds.), Handbook of research synthesis and meta-analysis ( ${ }^{\text {rd }}$ ed.), (pp. 3-16). Russell Sage Foundation.

Crandall, C. S. \& Sherman, J. W. (2016). On the scientific superiority of conceptual replications for scientific progress. Journal of Experimental Social psychology, 66, 9399.

Cronbach, L. J. (1975). Beyond the two disciplines of scientific psychology. American Psychologist, 30, 116-127,

Cummins, R, (2000). “How does it work?' versus “What are the laws?” Two conceptions of explanation. In F. C Keil \& R. A. Wilson (Eds.), Explanation and cognition (pp. 117144). MIT Press. 
Devezer, B., Navarro, D. J., Vandekerckhove, J., Buzbas, E. O. (2020). The case for formal methodolgy in scientific reform. bioRxiv. https://doi.org/10.1101/2020.04.26.048306

Earp, B. D., \& Trafimow, D. (2015). Replication, falsification, and the crisis of confidence in social psychology. Frontiers in Psychology. doi 10.3389/fpsyg.2105.00621.

Fanelli, D. (2018). Is science really facing a reproducibility crisis, and do we need to? Proceedings of the National Academy of Sciences. doi/10.1073/pnas.1708272114.

Fisk, D. W., \& Campbell, D. T. (1992). Citations do not solve problems. Psychological Bulletin, 112, 393-395.

Franklin, A. (1990). Experiment, right or wrong. Cambridge University Press.

Fidler, F., Chee, Y. E., Wintle, B. C., Burgman, M. A., McCarthy, M. A., \& Gordon, A. (2017). Metaresearch for evaluating reproducibility in ecology and evolution. BioScience, 67, 282-289.

Fidler, F., \& Wilcox, J. (2018). The reproducibility of scientific results. Stanford encyclopedia of philosophy (Winter 2018 Edition). E. N. Zolta (ed.). https://plato.stanford.edu/archives/win2018entries/scientific-reproducibility/.

Flynn, J. R. (2009). What is intelligence? Beyond the Flynn effect. (2 ${ }^{\text {nd }}$ ed.). Cambridge University press.

Gelman, A. (2018). The competing narratives of scientific revolution. https://ststsmodeling.stat,columbia.edu/2018/08/20.

Goldman, A. I., \& Beddor, B. (2015). Reliabilist epistemology. Stanford Encyclopedia of Philosophy (Winter 2018 Edition). E. N. Zolta (ed.). https://plato.stanford. edu/archives/win2018entries/reliabilist-epistemology/.

Guttinger, S. (2019). A new account of replication in the experimental life sciences. Philosophy of Science, 86, 453-471.

Guttinger, S. (2020). The limits of replicability. European Journal for Philosophy of Science, 10, $1-17$.

Haig, B. D. (2006). Consistency tests establish empirical generalizations. Nature, 443, 632.

Haig, B. D. (2011). Philosophical naturalism and scientific method. Psychological Inquiry, 22, 128-136.

Haig, B. D. (2013). Detecting psychological phenomena: Taking bottom up research seriously. American Journal of Psychology, 126, 135-153.

Haig, B. D. (2014). Investigating the psychological world: Scientific method in the behavioral sciences. MIT Press. 
Haig, B. D. (2017). Tests of statistical significance made sound. Educational \& Psychological Measurement, 77, 489-506.

Hardwicke, T. E., Serghiou, S., \& Janiaud, P., et al. (2020). Calibrating the scientific ecosystem through meta-research. Annual Review of Statistics and Its Application, 7, $11-37$.

Hedges, L. V., \& Schauer, J. M. (2019). More than one replication study is needed for unambiguous tests of replication. Journal of Educational and Behavioral Statistics, 44, 543-570.

Hubbard, R. (2016). Corrupt research: The case for reconceptualizing empirical management and social science. Sage.

Hull, D. (1988). Science as a process: An evolutionary account of the social and conceptual development of science. University of Chicago Press.

Ioannidis, J. P. A., Fanelli, D., Drake Dunn, D., \& Goodman, S. N. (2015). Meta-research: Evaluation and improvement of research methods and practices. PLOS Biology, 13 (10): e1002264.

Irzik, G., \& Nola, R. (2010). A family resemblance approach to the nature of science for science education. Science \& Education, 20, 591-607.

Jasny, B. R., Chin, G., Chong, L., \& Vignieri, S. (2011). Again, and again, and again ... . Science, 334, 1225.

Köhler, T., \& Cortina, J. M. (2020). Play it again, Sam! An analysis of constructive replication in the organizational sciences. Journal of Management, 20 (in press).

Kuhn, T. S. (1962/1970). The structure of scientific revolutions. University of Chicago Press.

Kuhn, T. S. (2000). The road since Structure. J. Conant \& J. Haugeland (Eds.). University of Chicago Press.

Kuorikoski, J., \& Marchionni, C. (2016). Evidential diversity and the triangulation of phenomena. Philosophy of Science, 83, 227-247.

Laudan, L. (1981). Science and hypothesis: Historical essays on scientific methodology. Reidel.

LeBel, E. P., \& Peters, K. R. (2011). Fearing the future of empirical psychology: Bem's (2011) evidence of psi as a case study of deficiencies in modal research practice. Review of General Psychology, 15, 371-379.

LeBel, E. P., Berger, D., Campbell, L., \& Loving, T. J. (2017). Falsifiability is not optional. Journal of Personality and Social Psychology, 113, 254-261. 
Leonelli, S. (2018). Re-thinking reproducibility as a criterion for research quality. Research in the History of Economic Thought and Methodology, 36B, 129-146.

Lycan, W. G. (1988). Judgement and justification. Cambridge University Press.

Lykken. D. T. (1968). Statistical significance in psychological research. Psychological Bulletin, 70, 151-159.

Lynch, J. G., Bradlow, E. T., Huber, J. C., \& Lehmann, D. R. (2015). Reflections on the replication corner: In praise of conceptual replications. International Journal of Research in Marketing, 32, 333-342.

Machery, E. (2020). What is a replication? Philosophy of Science. https://doi.org/10.1086/709701.

Machery, E., Grau, C., \& Pury, C. L. (2020). Love and power: Grau and Purdy (2014) as a case study in the challenges of X-phi replication. Review of Philosophy and Psychology, 5, 155-168.

Mahner, M. (2013). Science and pseudoscience: How to demarcate after the (alleged) demise of the demarcation problem. In M. Pigliucci \& M. Boudry (Eds.), Philosophy of pseudoscience: Reconsidering the demarcation problem (pp. 29-43). University of Chicago Press.

Makel, M. C., Plucker, J. A., \& Hegarty, B. (2012). Replications in psychological research: How often do they really occur? Perspectives on Psychological Science, 7, 537-542.

Maxwell, S. E., Lau, M. Y., \& Howard, G. S. (2015). Is psychology suffering from a replication crisis? What does "failure to replicate" really mean? American Psychologist, 70, 487-498.

Mayo, D. G. (1996). Error and the growth of experimental knowledge. University of Chicago Press.

Mayo, D. G. (2018). Statistical inference as severe testing: How to get beyond the statistics wars. Cambridge University Press.

Mayo, D. G., \& Spanos, A. (2011). Error statistics. In P. S. Bandyopadhyay \& M. R. Forster (Eds.), Handbook of philosophy of science, Vol. 7: Philosophy of statistics (pp. 153198). Elsevier.

Meehl, P. E. (1967). Theory-testing in psychology and physics: A methodological paradox. Philosophy of Science, 34, 103-115.

Mulkay, M., \& Gilbert, G. N. (1981). Putting philosophy to work: Karl Popper's influence on scientific practice. Philosophy of Social Science, 11, 389-407. 
Munafò, M. R., \& Smith, G. D. (2018). Repeating experiments is not enough. Nature, 553, 399-401.

Munafò, M. R., Nosek, B. A., Bishop, D. V. M., Button, K. S., Chambers, C. D., Percie du Sert, N., Simonsohn, U., Wagenmakers, E.-J., Ware, J. J., \& Ioannidis, J. P. A. (2017). A manifesto for reproducible science. Nature Human Behavior, 1 (21), 1-9.

Nelson, L. D, Simmons, J., \& Simonsohn, U. (2018). Psychology's renaissance. Annual Review of Psychology, 69, 511-534.

Nickles, T. (2017). Scientific revolutions. Stanford encyclopedia of philosophy (Winter 2018 Edition). E. N Zoltan (ed.), <https://plato.stanford.edu/archives/win2017/entries/scientific-revolutions/>

Nosek, B. A., Spies, J. R., \& Motyl, M. (2012). Scientific utopia II. Restructuring incentives and practices to promote truth over publishability, Perspectives on Psychological Science, 7, 615-631.

Nosek, B. A., \& Errington, T. M. (2017). Making sense of replications. eLife, 6:e23383.

Nosek, B. A., \& Errington, T. M. (2020). What is replication? PLOS Biology, 18 (3): e3000691

Nosek, B. A., \& Errington, T. M. (2020b). Argue about what a replication means before you do it. Nature, 583, 518-520.

Open Science Collaboration (2012). Estimating the reproducibility of psychological science, Science, 349, aac4716: 1-8.

Orlitzy, M. (2102). How can significance tests be institutionalized? Organizational Research Methods, 15, 199-228.

Pashler \& Harris (2012). Is the replicability crisis overblown? Three arguments examined. Psychological Science, 7, 331-536.

Popper, K. R. (1959). The logic of scientific discovery. Hutchinson.

Popper, K. R. (1969). Conjectures and refutations: The growth of scientific knowledge ( $3^{\text {rd }}$ ed.). Routledge \& Kegan Paul.

Reid, C. (1998). Neyman. Springer.

Roediger, H. L. (2012). Psychology's woes and a partial cure: The values of replication. Observer, 25, 1-7.

Salmon, W. C. (1984). Scientific explanation and the causal structure of the world. Princeton University Press.

Schickore, J. (2011). The significance of re-doing experiments: A contribution to historically informed methodology. Erkenntnis, 75, 325-374. 
Schmidt, F. L. (1992). What do data really mean? Research findings, meta-analysis, and cumulative knowledge in psychology. American Psychologist, 47, 1173-1181.

Schmidt, F. L. (1993). Meta-analysis and cumulative knowledge. Contemporary Psychology, $38,1163-1165$.

Schmidt, S. (2009). Shall we really do it again? The powerful concept of replication is neglected in the social sciences. Review of General Psychology, 13, 90-100.

Scholl, B. J. (2017). Reliability in psychology: Means versus ends. APS Observer, 30, 38-39.

Shiffrin, R. M., Börner, K., \& Stigler, S. M. (2018). Scientific progress despite irreproducibility: A seeming paradox. Proceedings of the National Academy of Sciences, 115, 2632-2639.

Sidman, M. (1960). Tactics of scientific research: Evaluating experimental data in psychology. Basic Books.

Simon, H. A. (1977). Models of discovery and other topics in the methods of science. Reidel.

Simons, D. J. (2014). The value of direct replication. Perspectives on Psychological Science, 9, 76-80.

Spellman, B. A. (2015). A short (personal) future history of revolution 2.0. Perspectives on Psychological Science, 10, 886-899.

Steinle, F. (2016). Stability and replication of experimental results: A historical perspective. In H. Atmanspacher \& S. Maasen (Eds.), Reproducibility: Principles, problems, practices, and prospects (pp. 39-63). John Wiley \& Sons.

Stroebe, W., \& Strack, F. (2014). The alleged crisis and the illusion of exact replication. Perspectives on Psychological Science, 9, 59-71.

Thagard, P. (1988). Computational philosophy of science. MIT Press.

Thagard, P. (1992). Conceptual revolutions. Princeton University Press.

Trahan. L., Stuebing, K. K., Hiscock, M. K., \& Fletcher, J. M. (2014). The Flynn effect: A meta-analysis. Psychological Bulletin, 140, 1332-1360

Uchino, B. N., Thoman, D., \& Byerly, S. (2010). Inference patterns in theoretical social psychology. Looking back as we move forward. Social and Personality Psychology Compass, 4, 417-427.

Van Rooij, I., \& Baggio, G. (2020). Theory before the test: How to build high-verisimilitude explanatory theories in psychological science. Perspectives on Psychological Science (in press).

Wimsatt, W. C. (2007). Re-engineering philosophy for limited beings. Piecewise approximations to reality. Harvard University Press. 
Woodward, J. (1989). Data and phenomena. Synthese, 79, 393-472.

Zwaan, R. A., Pecher, D., Paolacci, G., Bouwmeester, S., Verkoeijen, P., Dijkstra, K., \& Zeelenberg, R. (2018a). Participant nonnaiveté and the reproducibility of cognitive psychology. Psychonomic Bulletin \& Review, 25, 1968-1972.

Zwaan, R. A., Etz, A., Lucas, R. E., \& Donnellan, M. B. (2018b). Making replication mainstream. Behavioral and Brain Sciences, 41, 1-13. 